\title{
Weekday Dependence of German Stock Market Returns
}

\author{
by \\ Helmut Herwartz* \\ Institut für Statistik und Ökonometrie \\ Humboldt-Universität zu Berlin \\ Spandauer Str. 1 \\ 10178 Berlin \\ GERMANY
}

Tel.: (+30) 2093-5725

\begin{abstract}
The so-called 'Monday effect' has been found for various stock markets of the world. The empirical finding that Monday returns are significantly smaller than returns measured for the remaining days of the week calls the efficiency hypothesis for pricing processes operating on stock markets into question. Investigating an index series measured at the Frankfurt stock exchange the paper compares estimation results of parametric and nonparametric autoregressive models with respect to possible weekday dependence of return data. Allowing for heteroskedastic error distributions the wild bootstrap is used to infer against time varying means and correlation of return data in parametric models and to obtain confidence bands for nonparametric estimates. It is shown that time dependence is an important feature describing the dynamics of German stock market returns in the period 1960-79. Within two subsamples obtained from the period 1980-97 the evidence in favour of such effects is mitigated substantially.
\end{abstract}

Keywords: Periodic models, weekday effects, wild bootstrap, nonparametric autoregression JEL Classification C14, C22, G14

${ }^{*}$ The research of this paper was carried out within the Sonderforschungsbereich 373 at Humboldt University Berlin and was printed using funds made available by the Deutsche Forschungsgemeinschaft. Helpful comments from Michael Neumann and Rolf Tschernig are gratefully acknowledged. 


\section{Introduction}

Time dependent dynamics of stock market data have been detected in numerous empirical papers for various stock markets of the world. As the most popular seasonal feature of returns observed on stock markets one may regard the so-called Monday effect, describing that returns measured from Friday to Monday appear to be significantly negative in mean whereas positive returns are observed for the remaining days of the week. The Monday effect has a long history in the empirical literature (see e.g. Fields, 1931, Keim and Stambaugh, 1984) and calls the efficiency of financial markets into question. Without adjusting for risk the efficient market hypothesis essentially implies current prices to be the best forecasters of future prices. Pricing processes are expected to follow a so-called random walk possibly augmented with some deterministic drift term. Assuming prices of risky assets to follow a random walk with drift and taking calendar time into account it would appear quite natural to observe Monday returns to be on average 3 time larger than returns measured for the remaining days of the week. Thus the calendar time hypothesis is even more at odds with the magnitudes of average daily returns found in the empirical literature. For the German stock market Krämer and Runde (1996) investigate seasonal dynamics of daily returns of the German share price index DAX and most of its individual components. Comparing four subsamples obtained from their sample period 1960 to 1992 Krämer and Runde (1996) find the Monday effect to persist through time and to be of more importance for the index data in comparison to individual shares.

A further property of empirical returns is positive autocorrelation (see e.g. Campbell et al. (1997), Chapter 2). To some extent positive autocorrelation may be attributed to the presence of a time varying risk premium as discussed in Engle et al. (1987). In empirical practice, however, the fit of autoregressive models often outperforms the one of risk premium models (see e.g. Hafner and Herwartz 1999). From the viewpoint of a time series analyst the class of periodic time series models provides a general framework to detect time dependence of deterministic as well as stochastic properties of a time series under study. An essential feature of periodic models is to condition the data generating process of a time series variable on a deterministic function of time, for example on the season when analysing seasonal data or on the day of the week within an analysis of daily observations. In a number of papers periodic time series models are recommended for the analysis of seasonal data (see e.g. Cleveland and Tiao 1979, Tiao and Grupe 1980, Osborn 1991).

The issue of detecting periodic parameter variation is addressed in Lütkepohl (1992) where the likelihood principle is used within the framework of stationary vector autoregressive models. The asymptotic distribution of the likelihood ratio (LR-) statistic is derived under the assumption of underlying error terms which are independently and identically distributed (iid). Returns measured on financial markets, however, are known to exhibit volatility clustering, i.e. periods of lower price fluctuations and periods of higher volatility 
alternate. Time varying second order moments can be shown to involve considerable size distortions of test statistics derived under iid assumptions as for example the LR-statistic (see e.g. Herwartz 1998, Hafner and Herwartz 1999).

In the present paper the so-called wild bootstrap is used to infer against weekday effects for German stock market data. The wild bootstrap procedure copes conveniently with heteroskedasticity. In addition, nonparametric techniques are applied in order to give insight into the conditional and unconditional stochastic behaviour of German stock market returns. Compared to parametric models the latter approach provides a data driven framework to uncover important dynamic features which may remain undetected by parametric modeling.

The remainder of the paper is organized as follows: In Section 2 periodic autoregressive models and the LR-test against periodic parameter variation are briefly discussed. The wild bootstrap is outlined in Section 3. A parametric analysis of German stock market returns is provided in Section 4. The nonparametric autoregressive model is given in Section 5 also containing empirical results for German return data. Section 6 offers a brief discussion of the unconditional distributions of Monday vs. Friday returns observed on the German stock market. Section 7 summarizes and concludes the paper.

\section{Autoregressive Model Specifications}

Time dependent data generating processes are often employed to characterize seasonally varying data. The specification of time series models with seasonal dummy variables has become a standard tool to account for time varying means of an observed series. Within an even more general class of time series models, however, one may not only regard the expected value of a time series variable to depend on time but also second order moments, i.e. the autocovariance function. Models allowing for a time dependent autocovariance function have become popular as periodic time series models (see e.g. Box and Jenkins 1970). The class of autoregressive periodic models is easily introduced. In this case deterministic $\left(\nu_{s}\right)$ and autoregressive parameters $\left(\phi_{s, i}\right)$ are conditioned on a deterministic function of time $(s=s(t))$. Analysing seasonal data $s$ may indicate the season, turning to a daily sampling scheme $s$ may denote the day of the week. Given presample values $x_{0}, x_{-1}, \ldots, x_{1-p}$ a periodic autoregressive model of order $p$ can be given as follows:

$$
x_{t}=\nu_{s}+\phi_{s, 1} x_{t-1}+\phi_{s, 2} x_{t-2}+\cdots+\phi_{s, p} x_{t-p}+u_{t}, t=1, \ldots, T, s=1, \ldots, S .
$$

Applied to daily log-price changes observed on stock markets $(S=5)$ periodic time series models imply different return dynamics for each of the five trading days per week. The model in (1) provides a general framework to investigate dynamics of daily data. Further generalizations of the model in (1) are feasible. In particular, one may regard the autoregressive order or the error variance to be time dependent $\left(p=p_{s}\right.$ or $\left.\sigma^{2}=\sigma_{s}^{2}\right)$. Assuming all 
autoregressive parameters in (1) to be time invariant $\left(\phi_{s, i}=\phi_{i}, i=1, \ldots, p, s=1, \ldots, S\right)$ one obtains an autoregressive model with time depending first order moments. Given a particular autoregressive order the price to be paid for the flexibility of periodic models is the large number of parameters compared with a time invariant autoregressive process augmented with dummy variables to account for time dependent expectation.

A particular issue in specifying periodic time series models is to detect significant deviations from a time invariant model to avoid excess parameterization of an empirical model. The error sequence $u_{t}$ is assumed to be iid normally distributed $\left(u_{t} \sim N\left(0, \sigma^{2}\right)\right)$ to derive standard statistics as the LR-statistic for inference within a model like (1). Note that such an assumption may hardly be justified if financial market data are investigated. The effects of conditionally heteroskedastic error distributions introduced by Engle (1982) and generalized by Bollerslev (1986) on standard inference within periodic models will be of importance in Section 3.

The expectation of $x_{t}$ defined in (1) depends on $s$ and investigating the theoretical properties of $x_{t}$ somewhat deeper would reveal that also the autocovariance function $\gamma_{k}$,

$$
\gamma_{k}=\operatorname{cov}\left(x_{t}, x_{t-k}\right), k=0,1,2,3, \ldots,
$$

varies with $s=s(t)$, i.e. $\gamma_{k}=\gamma_{s, k}$. A time dependent autocovariance function violates the assumption of stationarity in the sense of invariant second order moments which is often made for the empirical analysis of time series data (see e.g. Osborn 1991). The process $x_{t}$, however, may be defined to be periodically stationary by means of the so-called vector representation which is related to (1). For this representation the observations which belong to an entire period are stacked into a $S$-dimensional vector, i.e. $\mathbf{x}_{\tau}=\left(x_{1, \tau}, \ldots, x_{S, \tau}\right)^{\prime}, \tau=1, \ldots, T / S$, and a vector autoregressive model is specified for $\mathbf{x}_{\tau}$ which is in line with (1).

Assuming $p=5$ to provide a reasonable autoregressive order for the analysis of daily data the univariate model in (1) yields the following vector representation:

$$
\begin{gathered}
\left(\begin{array}{ccccc}
1 & 0 & 0 & 0 & 0 \\
-\phi_{2,1} & 1 & 0 & 0 & 0 \\
-\phi_{3,2} & -\phi_{3,1} & 1 & 0 & 0 \\
-\phi_{4,3} & -\phi_{4,2} & -\phi_{4,1} & 1 & 0 \\
-\phi_{5,4} & -\phi_{5,3} & -\phi_{5,2} & -\phi_{5,1} & 1
\end{array}\right)\left(\begin{array}{l}
x_{1, \tau} \\
x_{2, \tau} \\
x_{3, \tau} \\
x_{4, \tau} \\
x_{5, \tau}
\end{array}\right)=\left(\begin{array}{l}
\nu_{1} \\
\nu_{2} \\
\nu_{3} \\
\nu_{4} \\
\nu_{5}
\end{array}\right) \\
+\left(\begin{array}{ccccc}
\phi_{1,5} & \phi_{1,4} & \phi_{1,3} & \phi_{1,2} & \phi_{1,1} \\
0 & \phi_{2,5} & \phi_{2,4} & \phi_{2,3} & \phi_{2,2} \\
0 & 0 & \phi_{3,5} & \phi_{3,4} & \phi_{3,3} \\
0 & 0 & 0 & \phi_{4,5} & \phi_{4,4} \\
0 & 0 & 0 & 0 & \phi_{5,5}
\end{array}\right)\left(\begin{array}{l}
x_{1, \tau-1} \\
x_{2, \tau-1} \\
x_{3, \tau-1} \\
x_{4, \tau-1} \\
x_{5, \tau-1}
\end{array}\right)+\left(\begin{array}{l}
u_{1, \tau} \\
u_{2, \tau} \\
u_{3, \tau} \\
u_{4, \tau} \\
u_{5, \tau}
\end{array}\right) .
\end{gathered}
$$

More compactly the model in (2) may be given as

$$
\Phi_{0} \mathbf{x}_{\tau}=\boldsymbol{\nu}+\Phi_{1} \mathbf{x}_{\tau-1}+\mathbf{u}_{\tau}
$$


In general the autoregressive orders of the univariate model in (1), $p$, and the vector autoregression in (3), $\tilde{p}$, are related as $\tilde{p}=1+[p / S]$, with [.] denoting 'the integer part of'. The series $x_{t}$ is defined to be periodically stationary if the vector series $\mathbf{x}_{\tau}$ is stationary. Let $z$ denote a complex variable. The vector process defined in (3) is stationary if the polynomial $\operatorname{det}\left(\Phi_{0}-\Phi_{1} z\right)$ has no roots in and on the complex unit circle (see e.g. Lütkepohl 1991), i.e.

$$
\left|\Phi_{0}-\Phi_{1} z\right|=0 \text { for }|z|>1 \text {. }
$$

In the case of stationarity the unconditional mean of $\mathbf{x}_{\tau}$ exists and can be given as:

$$
E\left[\mathbf{x}_{\tau}\right]=\left(\Phi_{0}-\Phi_{1}\right)^{-1} \boldsymbol{\nu}
$$

Obviously the elements of the mean vector $E\left[\mathbf{x}_{\tau}\right]$ may be time dependent even if in (1) deterministic components are time invariant $\left(\nu_{1}=\ldots=\nu_{S}=\nu\right)$. Within the class of periodic models a time varying expectation of a time series variable may be attributed to time dependence of autoregressive dynamics. For the detection of purely deterministic weekday effects in financial market data the analyst has to rule out periodic autoregressive dynamics. This is the reason why the broad class of periodic time series models is considered here as a convenient framework for the analysis of weekday dependencies of German stock market returns.

A key issue in empirical practice is raised in Tiao and Grupe (1980) and Osborn (1991). It can be shown that the 'unconditional autocovariance function' of a periodically stationary process,

$$
\gamma_{k}^{*}=\frac{1}{S} \sum_{s=1}^{S} \gamma_{s, k} k=0,1,2,3, \ldots,
$$

uniquely determines a nonperiodic autoregressive moving average (ARMA) model of appropriate order. Within the Box-Jenkins (1970) methodology empirical autocovariance functions have become a key means to identify ARMA-type processes. Ignoring the potential of periodicity, for example by computing and evaluating time invariant empirical autocovariance functions, the analyst runs the risk to misspecify a periodic time series process to be generated from a time invariant ARMA-representation.

For purposes like estimation or inference the model in (1) is often specified in terms of a reference 'season' $(s=1)$ :

$x_{t}=\nu+\phi_{1} x_{t-1}+\ldots+\phi_{p} x_{t-p}+\sum_{s=2}^{S} \nu_{s} D_{s, t}+\sum_{s=2}^{S} \phi_{s, 1} D_{s, t} x_{t-1}+\ldots+\sum_{s=2}^{S} \phi_{s, p} D_{s, t} x_{t-p}+u_{t}$. (5)

In (5) $D_{s, t}, s=2, \ldots, S$, denote dummy variables such that $D_{s, t}=1$ if $t$ belongs to season $s$ and $D_{s, t}=0$ otherwise. Note that a specification like (5) is helpful to infer against time dependent deviations from a nonperiodic time series model.

A number of standard inference techniques is available if the assumption of underlying Gaussian iid error terms $\left(u_{t}\right)$ is met or at least convenient. Lütkepohl (1992) proposes a 
couple of LR-statistics to test hypotheses of interest within a model like (1). Such hypotheses may be for example $H_{0}: \phi_{s, i}=\phi_{i}$ for all $i=1, \ldots, p$, or only some of them. A fully nonperiodic model is implied by $H_{0}: \nu_{s}=\nu, \phi_{s, i}=\phi_{i}, i=1, \ldots, p$. Under the assumption of Gaussian iid error terms with time invariant variance the LR-statistic is

$$
Q_{L R}=T \ln \left(\frac{\mathrm{RSS}_{r}}{\mathrm{RSS}_{u}}\right)
$$

with $\mathrm{RSS}_{r}$ and $\mathrm{RSS}_{u}$ denoting the ordinary Least Squares (OLS-) estimators of the residual sum of squared errors of the restricted and unrestricted model, respectively. $T$ indicates the number of observations in the sample. $Q_{L R}$ is asymptotically $\chi^{2}(k)$ distributed with $k$ denoting the number of excess parameters under the alternative hypothesis compared to the restricted model. The LR-statistic is a pivotal quantity since its asymptotic distribution does not depend on any unknown parameter.

Intuitively the LR-statistic compares the accuracy of fit obtained from a nested model relative to a more general specification. The loss of fit, measured in terms of $T\left(\ln \left(\mathrm{RSS}_{r}\right)-\right.$ $\ln \left(\mathrm{RSS}_{u}\right)$ ) should be insignificant if the restricted model is the true data generating process.

As mentioned, the asymptotic distribution of the LR-statistic in (6) depends on the assumption of underlying iid error terms, in particular $u_{t}$ is assumed to be homoskedastic. This assumption, however, is often violated in empirical practice. The variance of $u_{t}$ may depend on $s$ or vary across nonoverlapping partitions of an investigated sample period. In case of deterministic patterns of heteroskedasticity the LR-statistic in (6) can be conveniently modified in order to obtain an asymptotic $\chi^{2}$-distribution (see e.g. Lütkepohl 1992).

For the analysis of financial market data stochastic patterns of heteroskedasticity can be viewed as a predominant characteristic. Second order moments of financial market data are often specified in parametric form by means of the autoregressive conditionally heteroskedastic (ARCH-) process introduced by Engle (1982) and generalized by Bollerslev (1986) defining the GARCH-model. Within this framework the variance of $u_{t}$ is assumed to be governed by its own history and observed innovations $u_{t-1}, u_{t-2}, \ldots$. With $\omega$ denoting a deterministic component the $\operatorname{GARCH}(p, q)$ may be given as:

$$
u_{t} \sim N\left(0, \sigma_{t}^{2}\right), \sigma_{t}^{2}=\omega+\sum_{i=1}^{p} \alpha_{i} u_{t-i}^{2}+\sum_{i=1}^{q} \beta_{i} \sigma_{t-i}^{2}
$$

To ensure that the conditional variance, $\sigma_{t}^{2}$, is positive it is sufficient to assume that $\omega>$ $0, \alpha_{i} \geq 0$, and $\beta_{i} \geq 0$. $\alpha_{i}>0$ should hold for at least one $i=1, \ldots, p$. Since the introduction of the $\operatorname{ARCH}(p)$ model (i.e. the $\mathrm{GARCH}$ model with $q=0$ ) many theoretical and applied papers provided numerous alternative variance specifications which may be seen as complementary to the GARCH-model or as competing devices (see e.g. Engle and $\mathrm{Ng}$ 1993).

As defined above the $\operatorname{GARCH}(p, q)$ is time invariant. In the spirit of periodic processes one may also regard the variance generating process in $(7)$ to depend on $s$. The periodic 
GARCH-model is introduced in Bollerslev and Ghysels (1996), an early application of time dependent volatility processes can be found in Baillie and Bollerslev (1990). The present study concentrates on conditional mean estimation. Since the applied inference procedures are invariant with respect to the underlying process generating heteroskedastic error terms time varying volatility specifications are not further investigated.

GARCH-type error sequences complicate the specification of the likelihood function of a time series model like (5). Whereas Maximum Likelihood (ML-) estimation of the parameters in (7) governing variance dynamics always requires nonlinear optimization routines the parameters of the linear model in (5) may still be estimated by means of OLS-procedures. However, standard inference against significance of estimated autoregressive parameters in (5), say, is no longer valid. In the presence of conditionally heteroskedastic error terms the LR-statistic in (6) loses its asymptotic $\chi^{2}$-distribution and is no longer pivotal. Thus in the presence of conditional heteroskedasticity $Q_{L R}$ may be regarded as a pseudo LR-statistic.

Within a ML- or Quasi-ML (QML-) framework heteroskedasticity consistent inference by means of $t$-ratios requires to compute first and second order derivatives of the log-likelihood function with respect to the parameters of interest (see e.g. Bollerslev and Wooldridge 1992). A simultaneous analysis of linear and higher order dynamics might be cumbersome in applied work for a number of reasons. First, the performance of the nonlinear optimization may be negatively affected by large dimensions of the parameter space. In addition, increasing the number of parameters may reduce the power of inference procedures. Second, inference on linear dynamics within such a framework depends on the employed specification of the variance process. Since there is a variety of such specifications there is also a variety of $t$-ratios, say, that may be used to indicate significance of model parameters governing linear dynamics.

Due to these difficulties standard OLS-techniques are often used to estimate the conditional mean function of financial time series. In a second step of the analysis the interest turns to the specification of the variance process of estimated first step residuals. OLSestimation has a uniform solution which is straightforward to compute. However, inference along standard lines outlined above involves invalid empirical levels of tests derived under iid assumptions. Bootstrap inference may be seen as a means to retain the convenience of OLSprocedures and to overcome the invalidity of inference by mimicking the true distributions of particular LR-statistics for example. The wild bootstrap introduced by Wu (1986) accounts for heteroskedastic error distributions. Herwartz (1998) discusses the validity of wild bootstrap inference applied to the LR-statistic (6) for periodic time series models in the case of deterministic patterns of heteroskedasticity. In Herwartz and Hafner (1998) the performance of wild bootstrap inference is investigated in the presence of GARCH-type error terms. The wild bootstrap is recommended to mimic the distribution of the (pseudo) LR-statistic. Even in small samples $(T=50)$ heteroskedasticity consistent bootstrap inference is shown to pro- 
vide empirical size estimates which come close to their nominal counterparts. It is found that wild bootstrap inference applied to the LR-statistic has superior size properties compared to QML-inference in small samples and if QML-statistics are derived under misspecification of variance dynamics. Wild bootstrap inference is outlined in the next section.

\section{The Wild Bootstrap}

Wu (1986) introduced the wild bootstrap coping with heteroskedastic error distributions. Mammen (1993) proved this resampling scheme to work for $F$-type statistics in regression models with random explanatory variables. Neumann and Kreiss (1998) show that regression type bootstrap procedures remain valid for autoregression models if the underlying error terms are martingale difference sequences (mds), i.e. $E\left[u_{t} \mid u_{t-1}, u_{t-2}, \ldots\right]=0$. In the following the autoregressive model given in Section 2 is regarded as a regression model with random regressors. Since a resampling scheme is easy to implement under the null hypothesis of a nonperiodic data generating process the bootstrap might be seen as a promising alternative to iterative QML-inference procedures. Since the mds assumption appears to be reasonable for error terms of the linear model applied to financial market returns the wild bootstrap procedure is adopted to imitate the distribution of the (pseudo) LR-statistic given in (6). Suppose one is interested in testing the hypohesis $H_{0}: \phi_{s, i}=0, i=1, \ldots, p$, within a model like (5). Assuming presample values $x_{0}, x_{-1}, \ldots, x_{1-p}$ to be given an appropriate resampling procedure to mimic the distribution of the (pseudo) LR-statistic for this hypothesis may be given as follows:

1. The null hypothesis implies the following data generating process:

$$
\begin{aligned}
x_{t} & =\nu+D_{s, t} \nu_{s}+\phi_{1} x_{t-1}+\phi_{2} x_{t-2}+\cdots+\phi_{p} x_{t-p}+u_{t} \\
& =z_{0 t} \phi^{0}+u_{t} .
\end{aligned}
$$

In $(8)$ the row vector $z_{0 t}$ contains the set of explanatory variables under the null hypothesis and $\phi^{0}$ is an appropriate parameter vector. Without further distributional assumptions OLS provides a consistent estimate of the parameter vector $\left(\hat{\phi}^{0}\right)$, of the error terms $\left(\hat{u}_{0 t}\right)$ and of the (restricted) residual sum of squared errors $\left(\operatorname{RSS}_{r}=\sum_{t=1}^{T} \hat{u}_{0 t}^{2}\right)$ under the null hypothesis. Under the alternative hypothesis of periodically varying autoregressive parameters equation (8) is conveniently augmented obtaining:

$$
x_{t}=z_{0 t} \phi^{0}+z_{1 t} \phi^{1}+u_{t}
$$

In the present case the row vector $z_{1 t}$ in (9) contains lagged variables $x_{t-1}, \ldots, x_{t-p}$ multiplied by dummy variables $D_{s, t}$ as given in (5). The dimension of the parameter vector 
$\phi^{1}$ is equal to the number of excess parameters implied by the general model compared to the model under the null hypothesis, i.e. $k=(S-1) p$. OLS-estimation provides an estimate of the unrestricted residual sum of squared errors $\left(\operatorname{RSS}_{u}=\sum_{t=1}^{T} \hat{u}_{1 t}^{2}\right)$ and the LR-statistic in (6) can be calculated $\left(Q_{L R}\right)$.

2. The estimates $\hat{\phi}^{0}$ and $\hat{u}_{0 t}$ obtained under the null hypothesis are used to generate a wild bootstrap sample

$$
\tilde{x}_{t}=z_{0 t} \hat{\phi}^{0}+\tilde{u}_{t}, t=1, \ldots, T .
$$

Error terms $\tilde{u}_{t}$ are obtained from estimates $\hat{u}_{0 t}$ by mimicking their low order moments as follows. Imagine for each $t$ a random variable $\tilde{u}_{t}$ and a distribution $F_{t}$ such that

$$
E\left[\tilde{u}_{t} \mid F_{t}\right]=0, E\left[\tilde{u}_{t}^{2} \mid F_{t}\right]=\hat{u}_{0 t}^{2}, E\left[\tilde{u}_{t}^{3} \mid F_{t}\right]=\hat{u}_{0 t}^{3} .
$$

As a convenient specification of $F_{t}$ one may regard a two point distribution satisfying:

$$
\begin{aligned}
& \operatorname{prob}\left(\tilde{u}_{t}=(1-\sqrt{5}) \frac{\hat{u}_{0 t}}{2}\right)=\frac{(\sqrt{5}+1)}{2 \sqrt{5}} \\
& \operatorname{prob}\left(\tilde{u}_{t}=(1+\sqrt{5}) \frac{\hat{u}_{0 t}}{2}\right)=1-\frac{(\sqrt{5}+1)}{2 \sqrt{5}} .
\end{aligned}
$$

Since $\tilde{u}_{t}$ is obtained from a two point distribution it is easy to verify its low order moments. One obtains:

$$
E\left[\tilde{u}_{t}^{j}\right]=\left((1-\sqrt{5}) \frac{\hat{u}_{0 t}}{2}\right)^{j}\left(\frac{\sqrt{5}+1}{2 \sqrt{5}}\right)+\left((1+\sqrt{5}) \frac{\hat{u}_{0 t}}{2}\right)^{j}\left(1-\frac{\sqrt{5}+1}{2 \sqrt{5}}\right) .
$$

Solving (10) for $j=1,2,3$ it is seen that $\tilde{u}_{t}$ conveniently imitates the low order moments of $\hat{u}_{0 t}$, i.e.

$$
E\left[\tilde{u}_{t}\right]=0, E\left[\tilde{u}_{t}^{2}\right]=\hat{u}_{0 t}^{2}, E\left[\tilde{u}_{t}^{3}\right]=\hat{u}_{0 t}^{3} .
$$

Alternative procedures to generate wild bootstrap replicates $\tilde{u}_{t}$ are provided in Mammen (1993).

3. The generated random variables $\tilde{x}_{t}, t=1, \ldots, T$, are used to replace $x_{t}$ in (8) and (9), respectively. Analogously to step 1 the LR-statistic $\left(Q_{L R}^{*}\right)$ is computed for the generated series using the initial sets of explanatory variables $z_{0 t}$ and $z_{1 t}$.

4. Steps (2) and (3) are performed $R$ times with $R$ chosen sufficiently large. For each bootstrap sample the LR-statistic is recorded. For the experiments discussed in Section $4 R=1000$ was used.

5. The null hypothesis of interest is rejected with significance level $\alpha$ if $Q_{L R}$ exceeds the $(1-\alpha)$-quantile of $Q_{L R}^{*}$. 


\section{Detecting Time Dependence in German Stock Mar- ket Data}

\subsection{Data and parametric models}

In this section the inference procedures discussed before are applied to real financial data. Linear dynamics of the so-called DAFOX-series are investigated. This index is computed for research purposes by the Institute of Decision Theory and Business Research of the University of Karlsruhe, Germany (see Göppl and Schütz 1992). The DAFOX covers almost all stocks traded at the Frankfurt stock exchange and is conveniently adjusted for payments out of the stock. Therefore the following analysis concentrates on weekday dependence since other effects for example due to payments of dividends are ruled out by definition. The sample period is Monday, January-04-1960 to Tuesday, December-30-97 and contains 9499 observations. To obtain a stationary time series first differences of the logarithm of the index data are investigated. In order to provide a convenient framework for the comparison of the obtained results with Krämer and Runde (1996) the sample period is divided into 4 decades the last of which covers the period from January 1990 to December 1997. Empirical results for the entire sample period will be given for convenience.

To detect linear dependencies within the investigated series and to infer against deterministic weekday effects the following autoregressive model of order $p=5$ was employed with $x_{t}$ denoting current returns and $s=2, \ldots, 5$ indicating weekdays from Tuesday to Friday:

$$
x_{t}=\nu+\sum_{s=2}^{5} D_{s, t} \nu_{s}+\sum_{i=1}^{5} \phi_{i} x_{t-i}+u_{t} .
$$

From the literature on periodic time series models it is known that nonperiodic autoregressive time series specifications as (11) may have a similar (unconditional) autocovariance function as periodic models of lower autoregressive order (see e.g. Tiao and Grupe 1980). To allow for time dependence of the autoregressive part and to make sure that a periodic model has power against a nonperiodic specification the following time series model was applied complementary to (11):

$$
x_{t}=\nu+\sum_{s=2}^{5} D_{s, t} \nu_{s}+\phi_{1} x_{t-1}+\sum_{s=2}^{5} D_{s, t} \phi_{s, 1} x_{t-1}+\sum_{i=2}^{5} \phi_{i} x_{t-i}+u_{t} .
$$

\subsection{Empirical Results}

Estimation results for these models applied to the DAFOX data set are given in Table 1 (model (11)) and Table 2 (model (12)). To infer against significance of single estimated parameters appropriate LR-statistics are given jointly with their respective $\mathrm{p}$-values obtained from wild bootstrap resampling. Similarly LR-statistics testing against joint significance of 
selected parameters are given in Table 3 . In Tables 1 to 3 estimates which are significant at the $5 \%$ level are indicated with an asterisk. From the parametric time series approach the following results are obtained:

- Looking at the entire data set autoregressive dependencies cannot be rejected. An autoregressive model of order 2 is necessary to whiten estimated model errors when the AR(1)-parameters are allowed to be time varying. With respect to deterministic influences it turns out that the reference (Monday) intercept term $(\nu)$ is significantly less than zero and deterministic impacts $\left(\nu_{s}\right)$ measured for Wednesday, Thursday and Friday are significantly positive with the highest estimate obtained for Friday returns.

- $\mathrm{p}$-values obtained from the bootstrap procedure are in almost all cases greater than those obtained from a $\chi^{2}(1)$-distribution which would be the relevant asymptotic distribution of the LR-statistic in the presence of underlying iid error terms. Taking a significance level of $5 \%$ as a benchmark to reject a null hypothesis in question the bootstrap procedure yields different decisions relative to standard inference in a considerable number of cases. At the $5 \%$ significance level the critical value of LR-statistic is 3.84. Cumulating across four subsamples 5 (7) coefficient estimates which are found to be significant by standard inference for model (11) (model (12)) are insignificant according to the p-values obtained from the bootstrap procedure.

- The rejection of linear dependencies for financial market data supports the paradigm of efficiency. Using wild bootstrap inference which copes with heteroskedasticity in a convenient way mitigates the evidence in favour of higher autoregressive orders for all subsamples. For the last subsample linear dependencies are uniquely rejected irrespective of the empirical model employed for the analysis. With respect to the period 1980-89 a further interesting result is obtained: Taking the AR(5)-model as a framework for the empirical analysis all autoregressive parameters are found to be insignificant. Within the second specification, however, almost all time dependent AR(1)-parameters are found to be highly significant whereas higher order autoregressive parameters are not significant. Taking the literature on periodic time series models into account this result may be regarded as a recommendation in favour of a time dependent AR(1) time series model for DAFOX-returns in the period 1980-89.

- Summarizing the results of the inference exercises the hypothesis is supported that the stock market in Germany became more and more efficient in the course of time in the sense that DAFOX-returns became unpredictable by means of autoregressive time series models. Deterministic weekday effects are also found to die out through time. For the first three decades typical weekday dependencies are obtained at least partly for example negative mean returns on Monday or highest returns in mean on Friday. 
For the last subsample, however, deterministic effects that are significant at the $5 \%$ level are not obtained at all.

Regarding the results of tests against joint significance of parameters of interest similar conclusions as above are to be drawn. The hypothesis that current returns are completely unpredictable $\left(H_{0}: x_{t}=u_{t}\right)$ is rejected within both time series models for the first two decades under study. Turning to the two remaining subperiods the efficient market hypothesis cannot be rejected at the $5 \%$ significance level.

\section{Nonparametric Autoregression}

\subsection{Methodology}

Parametric autoregressive time series models as given in Section 2 essentially assume $x_{t}$ to be a linear function of the observed history $\left\{x_{t-1}, x_{t-2}, \ldots\right\}$. For applied work and especially for the analysis of financial market data linearity may be regarded as a strong restriction. In particular, the linear autoregressive model of order $p=1$ implies that positive and negative lagged returns have, on average, the same impact in absolute value on the current return of an asset. Such a symmetry hypothesis is hardly to justify by a-priori economic reasoning and a time series model designed for the analysis of empirical returns should at least allow to detect deviations from the standard linear model. Other nonlinearities for example size effects also bear some intuition for the time paths of empirical returns. A framework which is able to nest a wide range of relations between a random variable $X_{t}$ and its predecessor $X_{t-1}$ is the nonparametric autoregressive model of order one:

$$
X_{t}=m\left(X_{t-1}\right)+e_{t}, t=1, \ldots, T .
$$

In (13) $e_{t}$ denotes an error term which may be heteroskedastic satisfying

$$
E\left(e_{t} \mid X_{t-1}\right)=0, E\left(e_{t}^{2} \mid X_{t-1}\right)=v\left(X_{t-1}\right) .
$$

The conditional variance $v\left(X_{t-1}\right)$ is assumed to be finite. With respect to the conditional distribution of $e_{t}$ it is assumed that

$$
\mathcal{L}\left(e_{t} \mid X_{t-1}, X_{t-2}, \ldots, X_{0}\right)=\mathcal{L}\left(e_{t} \mid X_{t-1}\right)
$$

The (unknown) mean function $m(x)=E\left[X_{t} \mid X_{t-1}=x\right]$ and variance function $v(x)=$ $E\left[e_{t}^{2} \mid X_{t-1}=x\right]$ are conveniently estimated by nonparametric kernel estimators (see e.g. Tjøstheim 1994). Locally linear estimation (see e.g. Fan 1993) of autoregression functions is discussed in Masry (1996). The locally linear estimator of $m(x)$ is the first component of the solution of the following minimizing problem with respect to $m=\left(m_{0}, m_{1}\right)^{\prime}$ :

$$
\min _{m} Q(x)=\min _{m} \sum_{t=1}^{T} K\left(\frac{x-x_{t-1}}{h}\right)\left(x_{t}-m_{0}-m_{1} \frac{x-x_{t-1}}{h}\right)^{2}
$$


where $K($.$) is a symmetric kernel-function and h$ denotes the bandwidth parameter.

The estimated mean function can be given directly as a function of weighted observations with the weights depending on the applied kernel function, the bandwidth and the observations

$$
\begin{aligned}
\hat{m}(x) & =\sum_{t=1}^{T} w\left(x, h, x_{0}, \ldots, x_{T-1}\right) x_{t} \\
& =\left[\left(M_{x}^{\prime} K_{x} M_{x}\right)^{-1} M_{x}^{\prime} K_{x} X\right]_{1} .
\end{aligned}
$$

In (14) $X=\left(x_{1}, \ldots, x_{T}\right)^{\prime}$,

$$
M_{x}=\left(\begin{array}{cc}
1 & \left(x-x_{0}\right) / h \\
1 & \left(x-x_{1}\right) / h \\
\vdots & \vdots \\
1 & \left(x-x_{T-1}\right) / h
\end{array}\right), K_{x}=\operatorname{Diag}\left[K\left(\frac{x-x_{0}}{h}\right), K\left(\frac{x-x_{1}}{h}\right), \ldots, K\left(\frac{x-x_{T-1}}{h}\right)\right],
$$

and $[m]_{1}$ in (14) denotes the first element of the vector $m$. Due to the use of a kernel function the mean function of interest is always evaluated in a neighbourhood of the observed explanatory variables $x=x_{t-1}$. Therefore nonparametric estimates may be seen as a local average of the underlying mean function, implying that $\hat{m}(x)$ essentially estimates a smoothed version of $m(x)$. The magnitude of this bias increases with the bandwidth $h$. Choosing too small a bandwidth, however, results in a very wiggly pattern of $\hat{m}(x)$. Bandwidth selection is a key issue in nonparametric modeling (see e.g. Härdle et al. 1988). For the empirical analysis provided below the bandwidth parameter was determined by means of a so-called plug in selection rule which is shown in Ruppert et al. (1995) to converge conveniently fast to an optimal bandwidth solving the bias-variance trade-off mentioned before. A generalized version of this bandwidth selection procedure is implemented by Yang and Tschernig (1999) on the internet (http://www.blackwellpublishers.co.uk/rss).

The kernel estimate $\hat{m}(x)$ depends on the random sequence $e_{t}$. In order to characterize the stochastic behaviour of $\hat{m}(x)$ the time series analyst is often interested in computing confidence bands for the estimated autoregression function. While confidence bands for kernel estimates are straightforward to compute in the case of nonparametric regression, the framework of nonparametric autoregression poses some additional problems evolving from the interdependence of explanatory and dependent variables in (13) occurring in the course of time. Neumann and Kreiss (1998) show that the nonparametric autoregression model (13) can be treated analogously to the nonparametric regression model if a few assumptions concerning the sequence $X_{t}$ can be made reasonably. Apart from $\beta$-mixing conditions for $X_{t}$ it is assumed that $X_{t}$ is a strictly stationary Markov chain. The latter assumption may loosely be interpreted in such a way that within an autoregressive model of order one for example, $X_{t-1}$ conveys all information apart from $e_{t}$ which is necessary to characterize in mean the stochastic behaviour of $X_{t}$. This assumption may be related to the analysis of 
weekday dependence of stock market returns such that Tuesday returns for example are completely determined by Monday returns and some unpredictable influences occurring on Tuesday. The analysis provided by Neumann and Kreiss (1998) allows for a wide range of possible distributions of $e_{t}$ and is particularly valid in the presence of heteroskedastic error distributions characterizing returns measured on financial markets.

The construction of confidence bands for the estimated mean function $\hat{m}(x)$ with a prespecified significance level provides a number of interesting characteristics of the data set under study. First, the analyst gets some guidance in evaluating the adequacy of the periodic autoregressive model of order $p=1$. Such a model provides an adequate data generating process for empirical returns if it becomes possible to fit a line (e.g. estimates $\hat{x}_{t}$ implied by the linear model) within a confidence band with prespecified significance level over the entire support of explanatory variables $x=x_{t-1}$. Second, it is of interest whether such a line parallels the $x_{t-1}$-axis. A candidate constant value of $\hat{m}(x)$ may be the observed mean of $x_{t},\left(\hat{m}(x)=\bar{x}=1 / T \sum_{t=1}^{T} x_{t}\right)$, on the one hand or zero $(\hat{m}(x)=0)$ on the other. In the former case weekday effects are implied to be purely deterministic whereas the latter scenario supports the hypothesis that empirical returns cannot be forecasted by means of autoregressive models.

Confidence bands can be computed along the lines given in Neumann and Kreiss (1998). The stochastic behaviour of the estimator $\hat{m}(x)=\sum_{t=1}^{T} w\left(x, h, x_{0}, \ldots, x_{T-1}\right) x_{t}$ in (14) is analogue to the stochastic behaviour of $\hat{e}(x)=\sum_{t=1}^{T} w\left(x, h, x_{0}, \ldots, x_{T-1}\right) e_{t}$. The latter quantity is imitated by the wild bootstrap. Starting point for the bootstrap procedure is the estimation of model errors $\hat{e}_{t}=x_{t}-\hat{m}\left(x_{t-1}\right)$. The variance of $\hat{m}(x)$ is estimated as:

$$
\hat{v}(x)=\sum_{t}^{T} w^{2}\left(x, h, x_{0}, \ldots, x_{T-1}\right) \hat{e}_{t}^{2} .
$$

From the estimated errors it is straightforward to obtain wild bootstrap replicates as described in Section 3. In the following two approaches are distinguished: First the analyst may be interested in pointwise confidence intervals which have the assumed significance level for single points $x=x_{t-1}$. On the other hand one may be interested in a confidence band with valid significance level on a compact interval of observations $a \leq x=x_{t-1} \leq b$. Asymptotically valid procedures can be detailed as follows:

- Obtain $R$ wild bootstrap samples $\tilde{e}_{t}, t=1, \ldots, T$.

- Compute for each sample

$$
D^{*}(x)=\frac{\sum_{t=1}^{T} w\left(x, h, x_{0}, \ldots, x_{T-1}\right) \tilde{e}_{t}}{\sqrt{\hat{v}(x)}} .
$$

- 1. Pointwise confidence intervals at single points $x=x_{t-1}$ : Let $t_{\alpha}(x)$ denote the $(1-\alpha)$-quantile of $\left|D^{*}(x)\right|$. A confidence interval with asymptotically valid size 
for an interval around $x=x_{t-1}$ is obtained as:

$$
\hat{m}(x)-t_{\alpha}(x) \sqrt{\hat{v}(x)}, \hat{m}(x)+t_{\alpha}(x) \sqrt{\hat{v}(x)} .
$$

2. Simultaneously valid confidence bands for the entire range of $x=x_{t-1}$ : Let $t_{\alpha}$ denote the $(1-\alpha)$-quantile of

$$
\sup _{a \leq x \leq b}\left\{\left|D^{*}(x)\right|\right\}
$$

A confidence interval with asymptotically valid size for the autoregression function is obtained as:

$$
\hat{m}(x)-t_{\alpha} \sqrt{\hat{v}(x)}, \hat{m}(x)+t_{\alpha} \sqrt{\hat{v}(x)} \text {. }
$$

To apply the nonparametric estimation to DAFOX-returns the sample was divided into five subsamples for each day of the week. In contrast to the nonparametric estimation discussed above it was ensured that Tuesday returns $\left(x_{t}\right)$ for example are always explained by Monday returns $\left(x=x_{t-1}\right)$. Note that such a pairing of dependent and explanatory variables requires DAFOX measures at three consecutive trading days to be available. Nontrading days other than Saturday and Sunday cause some observations to be deleted from the sample.

The locally linear estimation was performed using the Gaussian kernel (see e.g. Silverman 1986):

$$
K(u)=\frac{1}{\sqrt{2 \pi}} \exp \left(-\frac{1}{2} u^{2}\right)
$$

As mentioned, the bandwidth parameter $h$ was selected using an automated plug-in selection rule. It turned out that the selected bandwidth depended heavily on the range of explanatory variables $x=x_{t-1}$. Boundary effects for bandwidth selection are discussed for example in Ruppert et al. (1995) advocating to estimate the function $m(x)$ on a compact interval of explanatory variables $\left[a \leq x=x_{t-1} \leq b\right]$ covering $100 \delta \%$ of all available observations, with $\delta$ chosen suitably small. An example illustrating boundary effects for bandwidth selection will be given below. To cope with boundary effects the nonparametric autoregression function was estimated for observed lagged returns between $-1.5 \%$ and $1.5 \%$. Depending on the investigated weekday and period this range covered between $87.95 \%$ (Tuesday return function in the period 1990-97) and 97.60\% (Monday return function in the period 1970-79) of all available observations (see Table 4). Instead of the "optimal" bandwidth $h_{\text {opt }}$ a smaller bandwidth, $0.8 h_{\text {opt }}$ was chosen in order to reduce to some extend the bias problem mentioned above. It turned out, however, that the obtained results did not differ qualitatively with respect to the choice of $h_{\text {opt }}$ or $0.8 h_{\text {opt }}$. 


\subsection{Empirical results}

To provide an illustrative example of the empirical nonparametric analysis Figure 1 shows the estimated mean function (solid line) of Monday returns, $\hat{m}_{\mathrm{mo}}(x)$, for the period 1960-69 which are assumed to depend on previous Friday returns. Including boundary explanatory variables the sample covers 463 observations and the selected optimal bandwidth is $h_{\text {opt }}=7.27 \mathrm{e}-03$. A $95 \%$ confidence band (dashed lines) which has asymptotically the valid size for the entire range of $x_{t-1}$ simultaneously (second approach given in Section 5.1) is shown jointly with $\hat{m}_{\text {mo }}(x)$. To make the nonparametric estimation problem more explicit the observed Monday returns are also displayed in the left hand panel of Figure 1. Note that providing single return coordinates $\left(x_{t-1}, x_{t}\right)$ may easily cause the scale of the graphs to be inconvenient. Therefore the results discussed in the remainder of this section concentrate on the estimated mean functions. To give some intuition of the implications inherent in the linear autoregressive model the implied OLS-estimates $\hat{x}_{t}$ are also provided (dotted line). A number of interesting features of the data set under study is obtained: the estimated mean-function is increasing in $x_{t-1}$ and apart from its behaviour at the boundaries almost linear. As one may expect the computed confidence bands are very close to $\hat{m}_{\mathrm{mo}}(x)$ in those regions where many observations are available and become wider for explanatory Friday returns which are large in absolute value and thus rarely observed. Due to the significant slope there is no meaningful constant (mean value of Monday returns $x_{t}$ or zero) falling into the computed confidence band for all observed values of Friday returns $x=x_{t-1}$. Comparing implied linear estimates and the obtained nonparametric confidence bands supports the linear AR(1)-model for this data set at the $5 \%$ significance level. All $\hat{x}_{t}$ computed from the linear model are contained in the estimated confidence band. Employing an undersmoothing bandwidth $0.8 h_{\text {opt }}$ instead of $h_{\text {opt }}$ almost identical results are obtained. The corresponding graphs are omitted here to economize on space. Results discussed below are obtained using a bandwidth $h=0.8 h_{\text {opt }}$.

As argued above the remaining empirical analysis adopting the nonparametric autoregressive model in (13) concentrates on typical ranges of observed explanatory variables, $-0.015 \leq x=x_{t-1} \leq 0.015$ for estimation and $-0.010 \leq x=x_{t-1} \leq 0.010$ with respect to graphical presentation of the results. To get some intuition on the relevance of these ranges Table 4 provides the relevant effective numbers of observations for each subsample and interval in absolute terms (obs.) and relative (rel.) to the number of available observations. For the data set discussed before (Monday return function, 1960-69) it turns out that within this subsample a bandwidth $h_{\text {opt }}=3.36 \mathrm{e}-03$ is selected. Neglecting about $5 \%$ of explanatory return variables at the margins reduces the optimal bandwidth by a factor of almost 0.5 for this particular sample. Therefore the following analysis investigates censored samples obtained from 'typical realisations' of explanatory return variables.

To characterize weekday effects in stock market returns it is now interesting to compare estimated return functions across weekdays and decades. The computed confidence bands 
provide an intuitively appealing benchmark to test informally for weekday dependence of return functions. The nonparametric framework allows for different time dependence of current returns for example with respect to negative and positive lagged returns. In addition, the analyst may get some impression whether weekday effects occur at the tails of the empirical distribution of $x_{t-1}$ (values of $x_{t-1}$ which are large in absolute value) or whether such effects are observed even in the center of this distribution (values of $x_{t-1}$ which are closer to zero). Table 4 also contains statistics of interest which are informally already introduced above: Conditional on the particular investigated range of $x_{t-1}$ the relative coverage of the linear model and two constants of interest, $\hat{m}(x)=\bar{x}$ and $\hat{m}(x)=0$, are given.

For the interval $-0.01 \leq x=x_{t-1} \leq 0.01$ Figure 2 and Figure 3 provide estimation results for consecutive decades. From Table 4 it is available that depending on the weekday and period of interest between $75.11 \%$ (Tuesday return function in the period 1990-97) and $87.48 \%$ (Friday return function in the period 1970-79) of all available observations fall into this range. Therefore weekday dependence as discussed in the following is an important characteristic for typical realisations of daily returns. The following results are obtained:

- The estimated mean return functions show considerable nonlinearties for most of the investigated 20 samples. On the $5 \%$ significance level, however, only for the Wednesday return function in the period 1960-69 implied OLS-estimates $\hat{x}_{t}$ are not entirely covered by the estimated nonparametric confidence bands. In this case $72.9 \%$ of estimated returns implied by the parametric model are contained in the nonparametric confidence band. For all remaining samples the nonparametric model supports the parametric specification in the sense that implied returns are contained in the $95 \%$ confidence band of the nonparametrically estimated function.

- With respect to the first two decades all nonparametric estimates exhibit significant slopes such that none of the investigated constant values is within the estimated confidence bands for the entire interval $-0.01 \leq x_{t-1} \leq 0.01$. Confidence bands of the Monday return function for example contain zero returns for only $27.6 \%$ (1960-69) and $20.8 \%$ (1970-79) of observed variables $x_{t-1}$. Turning to the two remaining investigated sample periods the corresponding statistics are $70.3 \%(1980-89)$ and $73.7 \%$ (1990-97) indicating that empirical returns have become more and more unpredictable in the course of time. Note that an analogous result was already obtained from the parametric approach in Section 4.

- For the last subsample period (1990-97) three of five estimated mean functions exhibit negative slopes for large (positive) values of explanatory return variables. Note that the second half of the period 1990-97 is characterized by a marked upward trending behaviour of stock prices which has been observed for numerous stock markets of industrial economies. The estimates given in the lower panels of Figure 3 might indicate 
the presence of 'technical' price adjustment in the sense that, on average, large positive lagged returns are followed by smaller or even negative price changes.

- For positive values of $x_{t-1}$ weekday dependencies of estimated mean return functions are not observed. Conditioning the analysis on negative values of lagged returns, however, weekday effects are observed comparing for example estimated Monday and Wednesday return functions in the period 1960-69. For lagged returns close to -0.01 the confidence bands obtained for these two return functions are disjoint such that one may regard $\hat{m}_{\text {mo }}(x)$ to be significantly less than $\hat{m}_{\text {we }}(x)$ for values of lagged returns in the neighbourhood of -0.01 . Similar conclusions can be drawn from a comparison of Monday and Friday returns in the period 1970-79. In the last but one decade under study mean Monday and Friday returns evaluated conditional on large negative lagged returns still appear to differ considerably but the estimated $95 \%$ confidence bands fail to be disjoint.

\section{Characterizing Unconditional Distributions}

Reviewing the results discussed in Section 5 and given in the empirical literature (see e.g. Krämer and Runde 1996) one may hold the view that a direct comparison of Monday vs. Friday stock market returns provides the strongest evidence in favour of weekday effects. From parametric models discussed in Section 4 the highest differences in estimated mean returns were obtained from a comparison of Monday and Friday returns for the first two decades. For the third decade under study Monday returns differ in mean almost equally from Friday and Wednesday returns. A priori the obtained differences may be attributed to a distribution of Friday returns which is completely located to the right of the distribution of Monday returns. However other scenarios may also generate the observed difference. For example a higher density of extremely negative Monday returns explains the same effect.

To characterize the unconditional distributions of Monday and Friday stock market returns kernel density estimates are discussed in the following. For kernel density estimation the observed sample is assumed to be independent and identically distributed. Taking the results reported above into account the iid assumption of empirical returns may be violated. Robinson (1983) proves the adequacy of kernel density estimation even for the case of dependent observations. Neumann (1998) proves that assuming some mixing conditions to hold the construction of confidence bands for kernel density estimates by means of iid-type bootstrap procedures is also valid in the case of weakly dependent (time series) data. Following Neumann (1998) the estimation and inference procedures adopted in this study can be detailed as follows:

- Assume a sample of possibly weakly dependent univariate random variables $\left(x_{1}, \ldots, x_{T}\right)$ 
to be available having unconditional density $f(x), x \in \mathbb{R}$. With $K(u)$ and $h$ denoting a kernel function and the bandwidth parameter, respectively, an estimator of $f(x)$ is:

$$
\hat{f}(x)=\frac{1}{T h} \sum_{t=1}^{T} K\left(\frac{x-x_{t}}{h}\right) .
$$

The variance of the density estimate in (16) can be estimated as:

$$
\hat{v}(x)=\frac{1}{T h^{2}}\left\{\frac{1}{T} \sum_{t}^{T} K^{2}\left(\frac{x-x_{t}}{h}\right)-\left[\frac{1}{T} \sum_{t}^{T} K\left(\frac{x-x_{t}}{h}\right)\right]^{2}\right\} .
$$

- Obtain from sampling with replacement $R$ bootstrap samples $\left(x_{1}^{*}, x_{2}^{*}, \ldots, x_{T}^{*}\right)$.

- Compute for each bootstrap sample a density estimate

$$
\hat{f}^{*}(x)=\frac{1}{T h} \sum_{t=1}^{T} K\left(\frac{x-x_{t}^{*}}{h}\right)
$$

and obtain

$$
D^{*}(x)=\left|\hat{f}(x)-\hat{f}^{*}(x)\right| / \sqrt{\hat{v}(x)}
$$

- 1. Confidence intervals at single points $x$ : Let $t_{\alpha}(x)$ denote the $(1-\alpha)$-quantile of $D^{*}(x)$. A confidence interval with asymptotically valid size within a small interval around $x$ is obtained as:

$$
\hat{f}(x)-t_{\alpha}(x) \sqrt{\hat{v}(x)}, \hat{f}(x)+t_{\alpha}(x) \sqrt{\hat{v}(x)} .
$$

2. Simultaneously valid confidence bands for the entire support of $x$ : Let $t_{\alpha}$ denote the $(1-\alpha)$-quantile of

$$
\sup _{x \in \mathbb{R}}\left\{D^{*}(x)\right\} .
$$

A confidence interval with asymptotically valid size is obtained as:

$$
\hat{f}(x)-t_{\alpha} \sqrt{\hat{v}(x)}, \hat{f}(x)+t_{\alpha} \sqrt{\hat{v}(x)} .
$$

Note that due to the application of kernel techniques again the estimated density functions are biased. For the computation of weekday return densities the bandwidth $h$ was chosen to be equal for Monday and Friday returns in order to avoid 'weekday' effects emerging from time dependent bandwidth selection. To characterize weekday dependence of unconditional returns informally confidence bands at single points $f(x)$ were constructed (first approach given above). With respect to the second approach (simultaneous confidence bands for the range of all observed returns) no significant differences between the estimated density functions were obtained at all. The density functions of interest were estimated and evaluated 
for a range of returns covering the interval $-0.03 \leq x \leq 0.03$ such that a few outliers were excluded from the sample. The optimal bandwidth $h$ was selected as the so-called rule-of-thumb bandwidth (see e.g. Silverman 1986), $h=1.06 \sigma_{x}^{-0.2}$, where $\sigma_{x}$ denotes the standard deviation obtained from a sample containing both, Monday and Friday returns. The Gaussian kernel given in (15) was employed for density estimation.

As shown in Figure 4 the estimated unconditional densities of all Monday and Friday returns observed in the period 1960-97 reveal only minor differences. Compared to the distribution of Monday returns (solid curve) the distribution of Friday returns (dashed curve) appears to be shifted somewhat to the right explaining that Friday returns are higher in mean than Monday returns. With respect to the first three investigated single decades (1960-89) essentially the same results are obtained. In the period 1990-97 large returns of either sign are more frequently observed on Mondays than on Fridays. Again only minor differences are observed for the estimated distributions. However for the first and last subsample small but positive returns have a considerably higher density for Friday returns in comparison to the Monday returns. Negative returns which are large in absolute value have a higher density in the distribution of Monday returns relative to the distribution of Friday returns. For positive returns of large absolute value an analogous result is not obtained, i.e. such returns have almost the same estimated densities for both trading days in all subsamples 1960-89. The $95 \%$ confidence intervals provided in Figure 5 show that at least the higher density associated with small positive values of Friday returns relative to Monday returns in the period 1960-69 is significant. For the remaining density estimates significant differences, measured in terms of nonoverlapping confidence bands, however, are not observed.

\section{Conclusions}

Depending on the period of interest German stock market returns show significant correlation. Allowing for time varying autoregressive dynamics periodic time series models provide a general framework to account for time dependent expectations. The wild bootstrap is adopted for inference in parametric models in the case of heteroskedastic errors which is relevant for the analysis of empirical return data. Estimation of the nonparametric autoregressive model is essentially data driven and provides evidence in favour of significant autocorrelation of German stock market returns.

Applying the nonparametric model the adequacy of linear return functions is called into question for 1 of 20 investigated subsamples (Wednesday return function in the period 196069). Weekday effects are shown to be present for typical ranges of lagged (explanatory) returns. Such effects measured by means of nonparametric confidence bands are of importance conditional on negative lagged returns and are not found if lagged returns are positive.

Weekday effects and autocorrelation of German return data are more evident within the 
first half of the investigated sample period (1960-79). Employing wild bootstrap inference within parametric autoregressive models for the period 1980-97 supports the hypothesis of completely unpredictable returns observed on the German stock market.

Investigating daily returns unconditionally by means of kernel density estimators yields only minor support for day dependent return distributions. Negative returns which are large in absolute value measured on Mondays have a higher estimated density compared to Friday returns.

\section{References}

1. R.T. Baillie, T. Bollerslev, 'Intra Day and Inter Market Volatility in Foreign Exchange Rates', Review of Economic Studies, 58, 565-585, (1990).

2. T. Bollerslev, 'Generalized Autoregressive Conditional Heteroskedasticity', Journal of Econometrics 31, 307-327, (1986).

3. T. Bollerslev, J.M. Wooldridge, 'Quasi Maximum Likelihood Estimation and Inference in Dynamic Models with Time Varying Covariances', Econometric Reviews, 11, 143$172,(1992)$.

4. A. Bose, 'Edgeworth Correction by Bootstrap in Autoregressions', The Annals of Statistics, 16, 1709-1722, (1988).

5. G.E.P. Box, G.M. Jenkins, Time Series Analysis, Forecasting and Control, Holden Day, San Francisco (1970).

6. J. Campbell, A. Lo, A. MacKinlay, The Econometrics of Financial Markets, Princeton University Press, Princeton, New Jersey, (1997).

7. W.P. Cleveland, G.C. Tiao, 'Modeling Seasonal Time Series', Revue Economic Appliquée, 32, 107-129, (1979).

8. R.F. Engle, 'Autoregressive Conditional Heteroscedasticity with Estimates of the Variance of U.K. Inflation', Econometrica, 50, 987-1008, (1982).

9. R.F. Engle, D.M. Lilien, R.P. Robins, 'Estimating the Varying Risk Premia in the Term Structure: the GARCH-M Model', Econometrica 55, 391-407, (1987).

10. R.F. Engle, V.K. Ng, 'Measuring and Testing the Impact of News on Volatility', Journal of Finance, 48, 1749-1778, (1993).

11. J. Fan, 'Local Linear Regression Smoothers and their Minimax Efficiency', The Annals of Statistics, 21, 196-216, (1993). 
12. M.J. Fields, 'Stock Prices: A Problem in Verification', Journal of Business, 4, 415-418, (1931).

13. T. Bollerslev, E. Ghysels, 'Periodic Autoregressive Conditional Heteroscedasticity', Journal of Business and Economic Statistics, 14, 139-151, (1996).

14. H. Göppl, H. Schütz, 'Die Konzeption eines Deutschen Aktienindex für Forschungszwecke (DAFOX)', Diskussionspapier 162, Institut für Entscheidungstheorie und Unternehmensforschung der Universität Karlsruhe, (1992).

15. W. Härdle, P. Hall, J.S. Marron, 'How Far are Automatically Chosen Regression Smoothing Parameters from their Optimum?' (with discussion), Journal of the American Statistical Association, 83, 83, 86-99, (1988).

16. H. Herwartz, 'Testing Periodicity in Time Series Models - A Recommendation of Bootstrap Methods', Computational Statistics, 13, 283-299, (1998).

17. C. Hafner, H. Herwartz, 'Testing for Linear Autoregressive Dynamics under Heteroskedasticity', Discussion Paper 7, SFB 373, Humboldt University Berlin, (1998).

18. D.B. Keim, R.F. Stambaugh 'A Further Investigation of the Weekend Effect in Stock Returns', Journal of Finance, 39, 819-840, (1984).

19. W. Krämer, R. Runde, 'Stochastic Properties of German Stock Returns', Empirical Economics, 21, 281-306, (1996).

20. H. Lütkepohl, Introduction to Multiple Time Series Analysis, Springer Verlag, Berlin, (1991).

21. H. Lütkepohl, 'Testing for Time Varying Parameters in Vector-Autoregressive Models', in: W.E. Griffiths, H. Lütkepohl, M.E. Bock (eds.), Readings in Econometric Theory and Practice, North-Holland, Amsterdam, 243-264, (1992).

22. E. Mammen, 'Bootstrap and Wild Bootstrap for High Dimensional Linear Models', The Annals of Statistics, 21, 255-285, (1993).

23. E. Masry, 'Multivariate Local Polynomial Regression for Time Series: Uniform Strong Consistency and Rates', Journal of Time Series Analysis, 17, 571-599, (1996).

24. M.H. Neumann, 'Strong Approximation of Density Estimators from Weakly Dependent Observations by Density Estimators from Independent Observations', The Annals of Statistics, 26, 2014-2048, (1998).

25. M.H. Neumann, J.P. Kreiss, 'Regression Type Inference in Nonparametric Autoregression, The Annals of Statistics, 26, 1570-1613, (1998). 
26. D.R. Osborn, 'The Implications of Periodically Varying Coefficients for Seasonal Time Series Processes', Journal of Econometrics, 28, 373-384, (1991).

27. P.M. Robinson, 'Nonparametric Estimators for Time Series', Journal of Time Series Analysis, 4, 185-207, (1983).

28. D. Ruppert, S.J. Sheather, M.P. Wand, 'An Effective Bandwidth Selector for Local Least Squares Regression', Journal of the American Statistical Association, 90, 12571270, (1995).

29. B.W. Silverman, Density Estimation for Statistics and Data Analysis, Chapman and Hall, London, (1986).

30. G.C. Tiao, M.R. Grupe, 'Hidden Periodic Autoregressive Moving Average Models in Time Series Data', Biometrika, 67, 365-373, (1980).

31. D. Tjøstheim, 'Nonlinear Time Series: A Selective Review', Scandinavian Journal of Statistics, 21, 97-130, (1994).

32. C.F.J. Wu, 'Jackknife, Bootstrap, and Other Resampling Methods in Regression Analysis' (with discussion), The Annals of Statistics, 14, 1261-1295, (1986).

33. L. Yang, R. Tschernig, 'Multivariate Bandwidth Selection for Local Linear Regression', Journal of the Royal Statistical Society, Ser. B, 61, forthcoming, (1999). 
Table 1: Estimation of the AR(5)-model with time varying expectation

\begin{tabular}{|c|c|c|c|c|c|c|c|c|c|c|}
\hline & \multicolumn{2}{|c|}{$60-69$} & \multicolumn{2}{|c|}{$70-79$} & \multicolumn{2}{|c|}{$80-89$} & \multicolumn{2}{|c|}{ 90-97 } & \multicolumn{2}{|c|}{$60-97$} \\
\hline & est. & LR & est. & LR & est. & LR & est. & LR & est. & LR \\
\hline$\nu$ & $-.49 \mathrm{e}-03$ & $\begin{array}{c}1.769 \\
(.24)\end{array}$ & $-.11 \mathrm{e}-02$ & $\begin{array}{l}11.23 \\
(.00)^{*}\end{array}$ & $-.45 \mathrm{e}-03$ & $\begin{array}{l}1.022 \\
(.42)\end{array}$ & $.52 \mathrm{e}-03$ & $\begin{array}{c}1.298 \\
(.36)\end{array}$ & $-.37 \mathrm{e}-03$ & $\begin{array}{c}3.394 \\
(.13)\end{array}$ \\
\hline$\nu_{2}$ & $-.46 \mathrm{e}-06$ & $\begin{array}{c}0.008 \\
(.93)\end{array}$ & $.12 \mathrm{e}-02$ & $\begin{array}{l}7.408 \\
(.00)^{*}\end{array}$ & $.49 \mathrm{e}-03$ & $\begin{array}{c}0.600 \\
(.50)\end{array}$ & $-.45 \mathrm{e}-03$ & $\begin{array}{c}0.509 \\
(.54)\end{array}$ & $.22 \mathrm{e}-03$ & $\begin{array}{c}0.635 \\
(.48)\end{array}$ \\
\hline$\nu_{3}$ & $.15 \mathrm{e}-02$ & $\begin{array}{l}8.753 \\
(.01)^{*}\end{array}$ & $.11 \mathrm{e}-02$ & $\begin{array}{l}6.573 \\
(.01)^{*}\end{array}$ & $.17 \mathrm{e}-02$ & $\begin{array}{l}6.962 \\
(.01)^{*}\end{array}$ & $.31 \mathrm{e}-03$ & $\begin{array}{c}0.240 \\
(.66)\end{array}$ & $.11 \mathrm{e}-02$ & $\begin{array}{l}16.45 \\
(.00)^{*}\end{array}$ \\
\hline$\nu_{4}$ & $.64 \mathrm{e}-03$ & $\begin{array}{c}1.545 \\
(.23)\end{array}$ & $.13 \mathrm{e}-02$ & $\begin{array}{l}8.093 \\
(.00)^{*}\end{array}$ & $.12 \mathrm{e}-02$ & $\begin{array}{c}3.624 \\
(.08)\end{array}$ & $.20 \mathrm{e}-03$ & $\begin{array}{c}0.100 \\
(.77)\end{array}$ & $.81 \mathrm{e}-03$ & $\begin{array}{l}8.281 \\
(.01)^{*}\end{array}$ \\
\hline$\nu_{5}$ & $.14 \mathrm{e}-02$ & $\begin{array}{l}7.454 \\
(.01)^{*}\end{array}$ & $.20 \mathrm{e}-02$ & $\begin{array}{c}19.41 \\
(.00)^{*}\end{array}$ & $.16 \mathrm{e}-02$ & $\begin{array}{l}6.614 \\
(.01)^{*}\end{array}$ & $-.94 \mathrm{e}-03$ & $\begin{array}{c}2.128 \\
(.20)\end{array}$ & $.11 \mathrm{e}-02$ & $\begin{array}{l}14.32 \\
(.00)^{*}\end{array}$ \\
\hline$\phi_{1}$ & .3126 & $\begin{array}{l}233.2 \\
(.00)^{*}\end{array}$ & .2277 & $\begin{array}{c}126.8 \\
(.00)^{*}\end{array}$ & .0635 & $\begin{array}{c}10.03 \\
(.26)\end{array}$ & .0273 & $\begin{array}{r}1.497 \\
(.44)\end{array}$ & .1394 & $\begin{array}{c}182.9 \\
(.00)^{*}\end{array}$ \\
\hline$\phi_{2}$ & -.1098 & $\begin{array}{l}27.40 \\
(.00)^{*}\end{array}$ & -.1091 & $\begin{array}{l}28.33 \\
(.00)^{*}\end{array}$ & -.0552 & $\begin{array}{c}7.565 \\
(.19)\end{array}$ & .0011 & $\begin{array}{c}0.003 \\
(.97)\end{array}$ & -.0517 & $\begin{array}{l}24.86 \\
(.01)^{*}\end{array}$ \\
\hline$\phi_{3}$ & .0182 & $\begin{array}{c}0.750 \\
(.53)\end{array}$ & .0592 & $\begin{array}{l}8.321 \\
(.04)^{*}\end{array}$ & .0149 & $\begin{array}{c}0.548 \\
(.71)\end{array}$ & .0261 & $\begin{array}{c}1.373 \\
(.48)\end{array}$ & .0208 & $\begin{array}{l}4.040 \\
(.25)\end{array}$ \\
\hline$\phi_{4}$ & .0074 & $\begin{array}{c}0.126 \\
(.80)\end{array}$ & .0505 & $\begin{array}{c}6.113 \\
(.06)\end{array}$ & .0121 & $\begin{array}{c}0.364 \\
(.73)\end{array}$ & -.0050 & $\begin{array}{c}0.051 \\
(.87)\end{array}$ & .0148 & $\begin{array}{c}2.040 \\
(.40)\end{array}$ \\
\hline$\phi_{5}$ & .0220 & $\begin{array}{c}1.213 \\
(.40)\end{array}$ & -.0393 & $\begin{array}{c}3.892 \\
(.21)\end{array}$ & .0323 & $\begin{array}{c}2.593 \\
(.32)\end{array}$ & -.0325 & $\begin{array}{c}2.122 \\
(.27)\end{array}$ & .0057 & $\begin{array}{c}0.313 \\
(.72)\end{array}$ \\
\hline
\end{tabular}

Estimation results (est.) and LR-statistics for the parametric model AR(5)-model with time invariant autoregressive parameters and weekday dependent deterministic influences $\left(x_{t}=\nu+\right.$ $\left.\sum_{s=2}^{5} D_{s, t} \nu_{s}+\sum_{i=1}^{5} \phi_{i} x_{t-i}+u_{t}\right)$. p-values obtained from wild bootstrap procedures in parentheses. * indicates significance at the $5 \%$ level. 
Table 2: Estimation of the AR(5)-model with time varying $\mathrm{AR}(1)$-parameter

\begin{tabular}{|c|c|c|c|c|c|c|c|c|c|c|}
\hline & \multicolumn{2}{|c|}{$60-69$} & \multicolumn{2}{|c|}{$70-79$} & \multicolumn{2}{|c|}{$80-89$} & \multicolumn{2}{|c|}{$90-97$} & \multicolumn{2}{|c|}{$60-97$} \\
\hline & est. & LR & est. & LR & est. & LR & est. & LR & est. & LR \\
\hline$\nu$ & $-.73 e-03$ & $\begin{array}{c}3.938 \\
(.08)\end{array}$ & $-.11 \mathrm{e}-02$ & $\begin{array}{l}13.08 \\
(.00)^{*}\end{array}$ & $-.81 e-03$ & $\begin{array}{c}3.306 \\
(.15)\end{array}$ & $.55 \mathrm{e}-03$ & $\begin{array}{c}1.443 \\
(.33)\end{array}$ & $-.51 \mathrm{e}-03$ & $\begin{array}{l}6.504 \\
(.04)^{*}\end{array}$ \\
\hline$\nu_{2}$ & $.19 \mathrm{e}-03$ & $\begin{array}{c}0.144 \\
(.71)\end{array}$ & $.13 \mathrm{e}-02$ & $\begin{array}{l}8.274 \\
(.00)^{*}\end{array}$ & $.77 e-03$ & $\begin{array}{c}1.518 \\
(.28)\end{array}$ & $-.48 \mathrm{e}-03$ & $\begin{array}{c}0.554 \\
(.53)\end{array}$ & $.34 \mathrm{e}-03$ & $\begin{array}{c}1.552 \\
(.28)\end{array}$ \\
\hline$\nu_{3}$ & $.17 \mathrm{e}-02$ & $\begin{array}{c}11.01 \\
(.00)^{*}\end{array}$ & $.12 \mathrm{e}-02$ & $\begin{array}{l}7.575 \\
(.00)^{*}\end{array}$ & $.20 \mathrm{e}-02$ & $\begin{array}{c}10.28 \\
(.00)^{*}\end{array}$ & $.29 \mathrm{e}-03$ & $\begin{array}{c}0.199 \\
(.68)\end{array}$ & $.12 \mathrm{e}-02$ & $\begin{array}{l}20.45 \\
(.00)^{*}\end{array}$ \\
\hline$\nu_{4}$ & $.90 \mathrm{e}-03$ & $\begin{array}{c}3.005 \\
(.10)\end{array}$ & $.14 \mathrm{e}-02$ & $\begin{array}{l}9.272 \\
(.00)^{*}\end{array}$ & $.15 \mathrm{e}-02$ & $\begin{array}{l}5.533 \\
(.03)^{*}\end{array}$ & $.21 \mathrm{e}-03$ & $\begin{array}{c}0.109 \\
(.78)\end{array}$ & $.96 \mathrm{e}-03$ & $\begin{array}{l}11.44 \\
(.00)^{*}\end{array}$ \\
\hline$\nu_{5}$ & $.16 \mathrm{e}-02$ & $\begin{array}{l}10.16 \\
(.00)^{*}\end{array}$ & $.25 \mathrm{e}-02$ & $\begin{array}{l}21.15 \\
(.00)^{*}\end{array}$ & $.20 \mathrm{e}-02$ & $\begin{array}{l}10.16 \\
(.00)^{*}\end{array}$ & $-.99 \mathrm{e}-03$ & $\begin{array}{c}2.336 \\
(.18)\end{array}$ & $.12 \mathrm{e}-02$ & $\begin{array}{c}18.29 \\
(.00)^{*}\end{array}$ \\
\hline$\phi_{1}$ & .5691 & $\begin{array}{l}133.7 \\
(.00)^{*}\end{array}$ & .3226 & $\begin{array}{l}55.81 \\
(.00)^{*}\end{array}$ & .3428 & $\begin{array}{l}48.60 \\
(.00)^{*}\end{array}$ & .1185 & $\begin{array}{c}4.829 \\
(.15)\end{array}$ & .3239 & $\begin{array}{l}173.6 \\
(.00)^{*}\end{array}$ \\
\hline$\phi_{2,1}$ & -.3384 & $\begin{array}{l}31.82 \\
(.02)^{*}\end{array}$ & -.1210 & $\begin{array}{l}4.057 \\
(.25)\end{array}$ & -.4032 & $\begin{array}{l}42.42 \\
(.01)^{*}\end{array}$ & -.1059 & $\begin{array}{c}2.461 \\
(.42)\end{array}$ & -.2610 & $\begin{array}{l}70.01 \\
(.00)^{*}\end{array}$ \\
\hline$\phi_{3,1}$ & -.3502 & $\begin{array}{l}29.31 \\
(.01)^{*}\end{array}$ & -.1361 & $\begin{array}{l}4.934 \\
(.18)\end{array}$ & -.3369 & $\begin{array}{l}25.93 \\
(.01)^{*}\end{array}$ & -.1332 & $\begin{array}{c}3.260 \\
(.37)\end{array}$ & -.2450 & $\begin{array}{l}53.51 \\
(.00)^{*}\end{array}$ \\
\hline$\phi_{4,1}$ & -.2748 & $\begin{array}{l}16.85 \\
(.03)^{*}\end{array}$ & -.1180 & $\begin{array}{c}3.768 \\
(.27)\end{array}$ & -.2297 & $\begin{array}{l}11.57 \\
(.09)\end{array}$ & -.1323 & $\begin{array}{c}3.062 \\
(.22)\end{array}$ & -.1860 & $\begin{array}{l}29.63 \\
(.01)^{*}\end{array}$ \\
\hline$\phi_{5,1}$ & -.2297 & $\begin{array}{l}12.99 \\
(.03)^{*}\end{array}$ & -.1028 & $\begin{array}{c}2.714 \\
(.34)\end{array}$ & -.3257 & $\begin{array}{l}22.48 \\
(.00)^{*}\end{array}$ & -.0632 & $\begin{array}{c}0.648 \\
(.56)\end{array}$ & -.1752 & $\begin{array}{l}26.13 \\
(.01)^{*}\end{array}$ \\
\hline$\phi_{2}$ & -.1168 & $\begin{array}{l}31.22 \\
(.00)^{*}\end{array}$ & -.1075 & $\begin{array}{l}27.51 \\
(.00)^{*}\end{array}$ & -.0490 & $\begin{array}{l}6.021 \\
(.27)\end{array}$ & .0008 & $\begin{array}{c}0.001 \\
(.98)\end{array}$ & -.0516 & $\begin{array}{l}25.00 \\
(.01)^{*}\end{array}$ \\
\hline$\phi_{3}$ & .0230 & $\begin{array}{l}1.208 \\
(.39)\end{array}$ & .0602 & $\begin{array}{l}8.606 \\
(.04)^{*}\end{array}$ & .0234 & $\begin{array}{c}1.363 \\
(.56)\end{array}$ & .0258 & $\begin{array}{l}1.332 \\
(.48)\end{array}$ & .0238 & $\begin{array}{c}5.303 \\
(.18)\end{array}$ \\
\hline$\phi_{4}$ & .0028 & $\begin{array}{c}0.018 \\
(.92)\end{array}$ & .0507 & $\begin{array}{l}6.182 \\
(.06)\end{array}$ & .0160 & $\begin{array}{c}0.643 \\
(.63)\end{array}$ & -.0041 & $\begin{array}{c}0.033 \\
(.90)\end{array}$ & .0151 & $\begin{array}{r}2.147 \\
(.37)\end{array}$ \\
\hline$\phi_{5}$ & .0197 & $\begin{array}{l}0.991 \\
(.46)\end{array}$ & -.0390 & $\begin{array}{c}3.804 \\
(.22)\end{array}$ & .0371 & $\begin{array}{c}3.487 \\
(.26)\end{array}$ & -.0348 & $\begin{array}{c}2.421 \\
(.23)\end{array}$ & .0051 & $\begin{array}{c}0.244 \\
(.75)\end{array}$ \\
\hline
\end{tabular}

Estimation results (est.) and LR-statistics for the parametric model AR(5)-model with time depending $\mathrm{AR}(1)$ parameter and deterministic components $\left(x_{t}=\nu+\sum_{s=2}^{5} D_{s, t} \nu_{s}+\right.$ $\left.\sum_{s=1}^{5} D_{s, t} \phi_{s, 1} x_{t-1}+\sum_{i=2}^{5} \phi_{i} x_{t-i}+u_{t}\right)$. p-values obtained from wild bootstrap procedures in parentheses. ${ }^{*}$ indicates significance at the $5 \%$ level. 
Table 3: Hypothesis Testing in parametric AR-models

\begin{tabular}{l|ccccc|cccccc}
\hline & \multicolumn{7}{|c|}{ Time invariant AR(5)-Model } & \multicolumn{3}{c}{ AR(5)-Model with $\phi_{s, 1}$ time dependent } \\
\hline Null hypothesis & $60-69$ & $70-79$ & $80-89$ & $90-97$ & $60-97$ & $60-69$ & $70-79$ & $80-89$ & $90-97$ & $60-97$ \\
\hline$H_{0}: \nu_{s}=0$ & 16.99 & 20.04 & 10.76 & 5.231 & 26.52 & 19.14 & 21.96 & 15.21 & 5.523 & 31.53 \\
& $(.00)^{*}$ & $(.00)^{*}$ & $(.03)^{*}$ & $(.26)$ & $(.00)^{*}$ & $(.00)^{*}$ & $(.00)^{*}$ & $(.00)^{*}$ & $(.26)$ & $(.00)^{*}$ \\
$H_{0}: \phi_{i}=0$ & 236.9 & 150.8 & 20.08 & 5.036 & 198.2 & 275.8 & 157.3 & 66.87 & 4.527 & 79.66 \\
& $(.00)^{*}$ & $(.00)^{*}$ & $(.44)$ & $(.84)$ & $(.00)^{*}$ & $(.00)^{*}$ & $(.00)^{*}$ & $(.19)$ & $(.77)$ & $(.00)^{*}$ \\
$H_{0}: x_{t}=u_{t}$ & 254.7 & 165.8 & 40.33 & 12.82 & 237.7 & 293.6 & 172.3 & 87.12 & 9.563 & 277.8 \\
& $(.00)^{*}$ & $(.00)^{*}$ & $(.16)$ & $(.63)$ & $(.00)^{*}$ & $(.00)^{*}$ & $(.00)^{*}$ & $(.09)$ & $(.91)$ & $(.00)^{*}$ \\
$H_{0}: \phi_{s, 1}=0$ & & & & & & 38.92 & 6.496 & 46.79 & 9.759 & 106.2 \\
& & & & & & $(.03)^{*}$ & $(.51)$ & $(.15)$ & $(.68)$ & $(.00)^{*}$ \\
$H_{0}: \nu_{s}=0, \phi_{s, 1}=0$ & & & & & & 55.91 & 26.54 & 57.55 & 17.35 & 317.4 \\
& & & & & & $(.01)^{*}$ & $(.07)$ & $(.10)$ & $(.80)$ & $(.00)^{*}$ \\
\hline
\end{tabular}

LR-statistics against joint significance of selected parameters within the investigated time series models. p-values obtained from bootstrap procedures in parentheses. ${ }^{*}$ indicates significance at the $5 \%$ level. 
Table 4: Coverage frequencies of nonparametric confidence bands

\begin{tabular}{|c|c|c|c|c|c|c|c|c|c|c|}
\hline & obs. & rel. & OLS & 0 & $\bar{x}$ & obs. & rel. & OLS & 0 & $\bar{x}$ \\
\hline & \multicolumn{10}{|c|}{$-0.015 \leq x=x_{t-1} \leq 0.015$} \\
\hline & \multicolumn{5}{|c|}{$\begin{array}{l}60-69 \\
\end{array}$} & \multicolumn{5}{|c|}{$70-79$} \\
\hline Mo & 439 & 0.95 & 1.00 & 0.30 & 0.21 & 448 & 0.99 & 1.00 & 0.27 & 0.29 \\
\hline $\mathrm{Tu}$ & 433 & 0.91 & 0.96 & 0.22 & 0.22 & 450 & 0.95 & 1.00 & 0.67 & 0.52 \\
\hline We & 437 & 0.93 & 0.77 & 0.57 & 0.29 & 454 & 0.96 & 1.00 & 0.45 & 0.40 \\
\hline Th & 443 & 0.93 & 0.99 & 0.55 & 0.69 & 455 & 0.96 & 1.00 & 0.76 & 0.74 \\
\hline \multirow[t]{2}{*}{ Fr } & 445 & 0.95 & 1.00 & 0.38 & 0.29 & 450 & 0.97 & 1.00 & 0.37 & 0.39 \\
\hline & \multicolumn{5}{|c|}{$80-89$} & \multicolumn{5}{|c|}{ 90-97 } \\
\hline Mo & 411 & 0.91 & 1.00 & 0.65 & 0.64 & 340 & 0.93 & 1.00 & 0.74 & 0.75 \\
\hline $\mathrm{Tu}$ & 442 & 0.93 & 1.00 & 1.00 & 0.97 & 336 & 0.88 & 1.00 & 0.89 & 1.00 \\
\hline We & 429 & 0.91 & 1.00 & 0.79 & 0.88 & 357 & 0.94 & 1.00 & 1.00 & 1.00 \\
\hline Th & 436 & 0.93 & 1.00 & 0.46 & 1.00 & 352 & 0.93 & 1.00 & 0.85 & 1.00 \\
\hline \multirow[t]{3}{*}{$\mathrm{Fr}$} & 417 & 0.91 & 1.00 & 0.80 & 0.86 & 346 & 0.93 & 1.00 & 0.98 & 0.99 \\
\hline & \multicolumn{10}{|c|}{$-0.010 \leq x=x_{t-1} \leq 0.010$} \\
\hline & \multicolumn{5}{|c|}{$60-69$} & \multicolumn{5}{|c|}{$70-79$} \\
\hline Mo & 399 & 0.86 & 1.00 & 0.28 & 0.20 & 391 & 0.85 & 1.00 & 0.21 & 0.28 \\
\hline $\mathrm{Tu}$ & 378 & 0.79 & 1.00 & 0.23 & 0.27 & 408 & 0.86 & 1.00 & 0.68 & 0.41 \\
\hline We & 377 & 0.80 & 0.73 & 0.55 & 0.24 & 403 & 0.86 & 1.00 & 0.46 & 0.42 \\
\hline Th & 398 & 0.84 & 1.00 & 0.55 & 0.53 & 404 & 0.85 & 1.00 & 0.85 & 0.89 \\
\hline \multirow[t]{2}{*}{$\mathrm{Fr}$} & 407 & 0.87 & 1.00 & 0.40 & 0.27 & 406 & 0.87 & 1.00 & 0.35 & 0.35 \\
\hline & \multicolumn{5}{|c|}{$80-89$} & \multicolumn{5}{|c|}{$90-97$} \\
\hline Mo & 364 & 0.80 & 1.00 & 0.70 & 0.72 & 303 & 0.83 & 1.00 & 0.74 & 0.85 \\
\hline $\mathrm{Tu}$ & 376 & 0.79 & 1.00 & 1.00 & 0.95 & 287 & 0.75 & 1.00 & 0.88 & 1.00 \\
\hline We & 377 & 0.80 & 1.00 & 0.83 & 0.95 & 313 & 0.83 & 1.00 & 1.00 & 1.00 \\
\hline Th & 371 & 0.79 & 1.00 & 0.50 & 0.93 & 308 & 0.81 & 1.00 & 0.83 & 1.00 \\
\hline Fr & 367 & 0.80 & 1.00 & 0.84 & 0.92 & 318 & 0.85 & 1.00 & 1.00 & 1.00 \\
\hline
\end{tabular}

Effective numbers (obs.) of available observations for complete samples and typical ranges of lagged returns. 'rel.' denotes 'obs.' relative to all available observations. 'OLS', ' 0 ', and ' $\bar{x}$ ' denote the relative number of sample points where the OLS-estimates of $x_{t}$, zero, and the (subsample) mean of $x_{t}$ is contained in a $95 \%$ confidence interval of the relevant mean return function. 

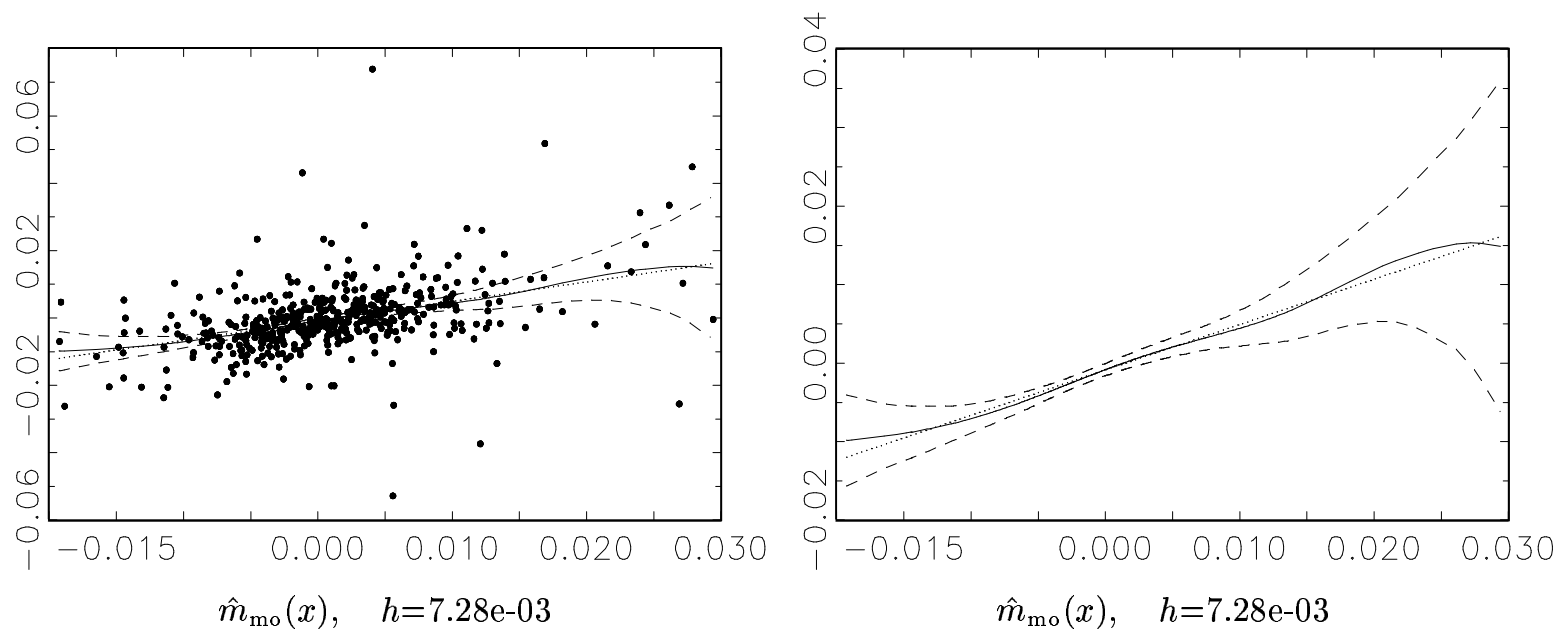

Figure 1: Estimated mean function for Monday returns $\left(\hat{m}_{\mathrm{mo}}(x)\right)$ (solid line) with simultaneously valid 95\% confidence bands (dashed lines), and OLS-estimates (dotted line) for the period 1960-69. 

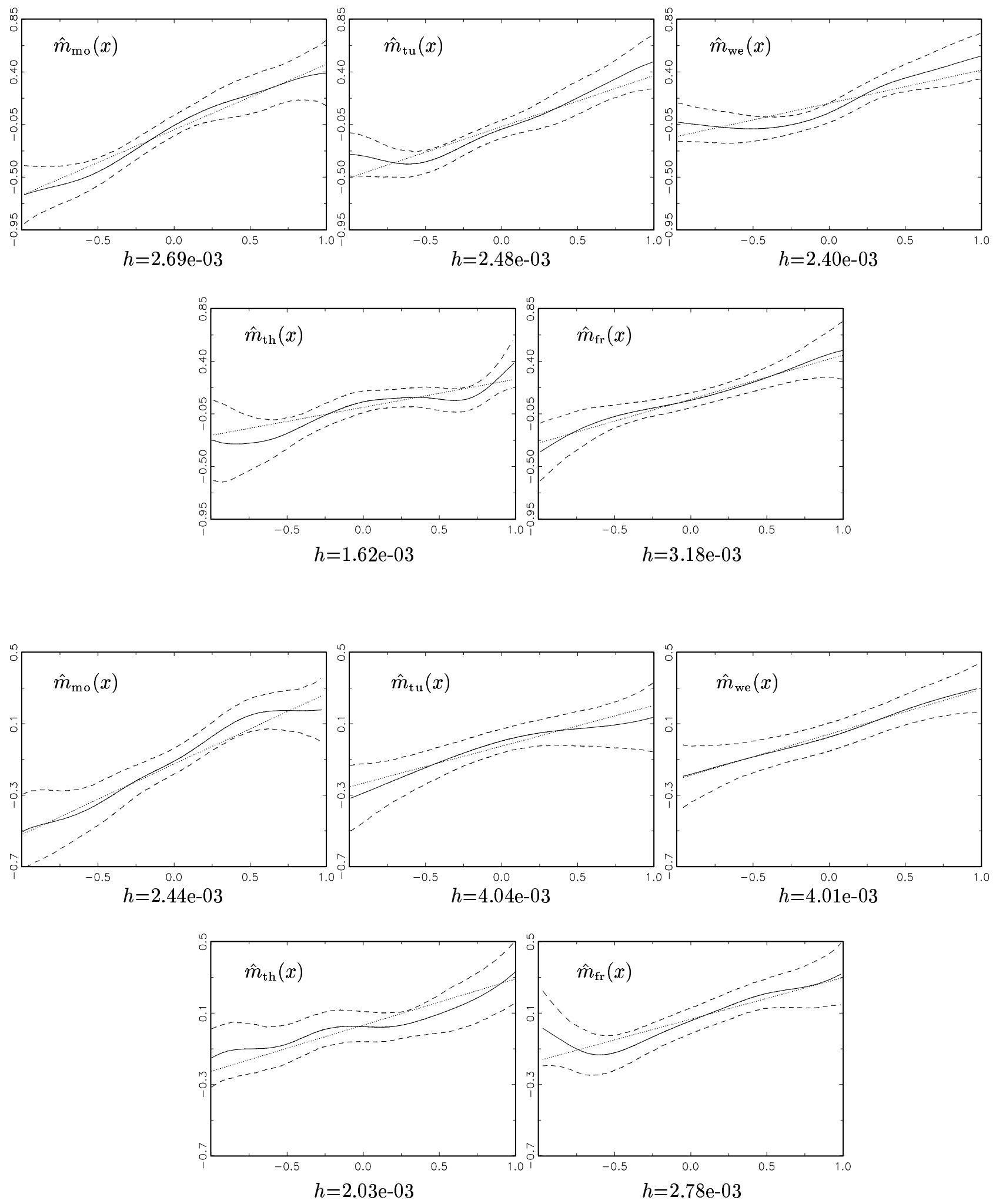

Figure 2: Estimated mean return functions, 95\% confidence bands, and OLS-estimates for 1960-69 (upper panels) and 1970-79 (lower panels). The relevant range of lagged returns is $-0.01 \leq x_{t-1} \leq 0.01$. The scale of both axes is multiplied by $10^{2}$. 

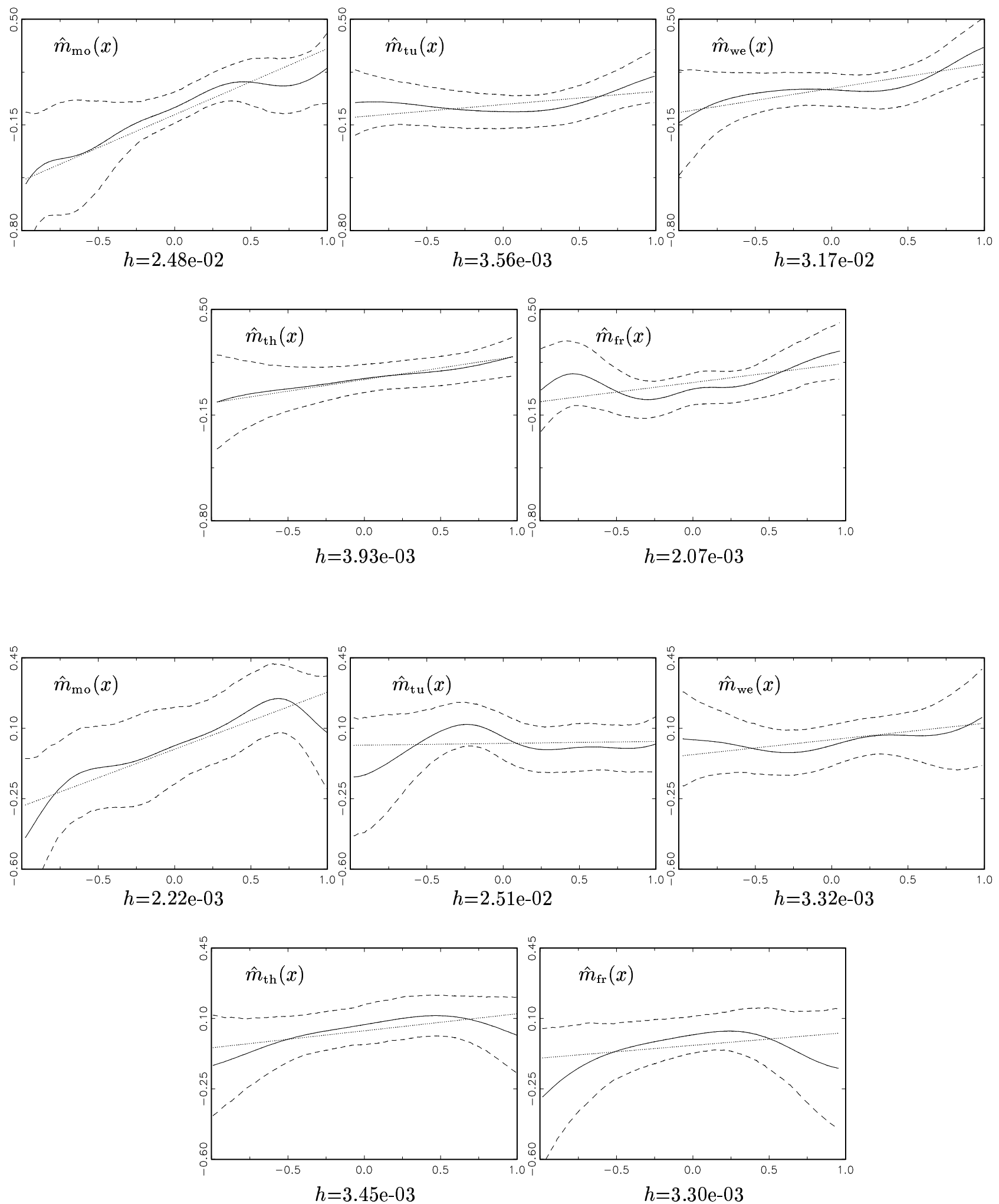

Figure 3: Estimated mean return functions, 95\% confidence bands and OLS-estimates for 1980-89 (upper panels) and 1990-97 (lower panels). The relevant range of lagged returns is $-0.01 \leq x_{t-1} \leq 0.01$. The scale of both axes is multiplied by $10^{2}$. 

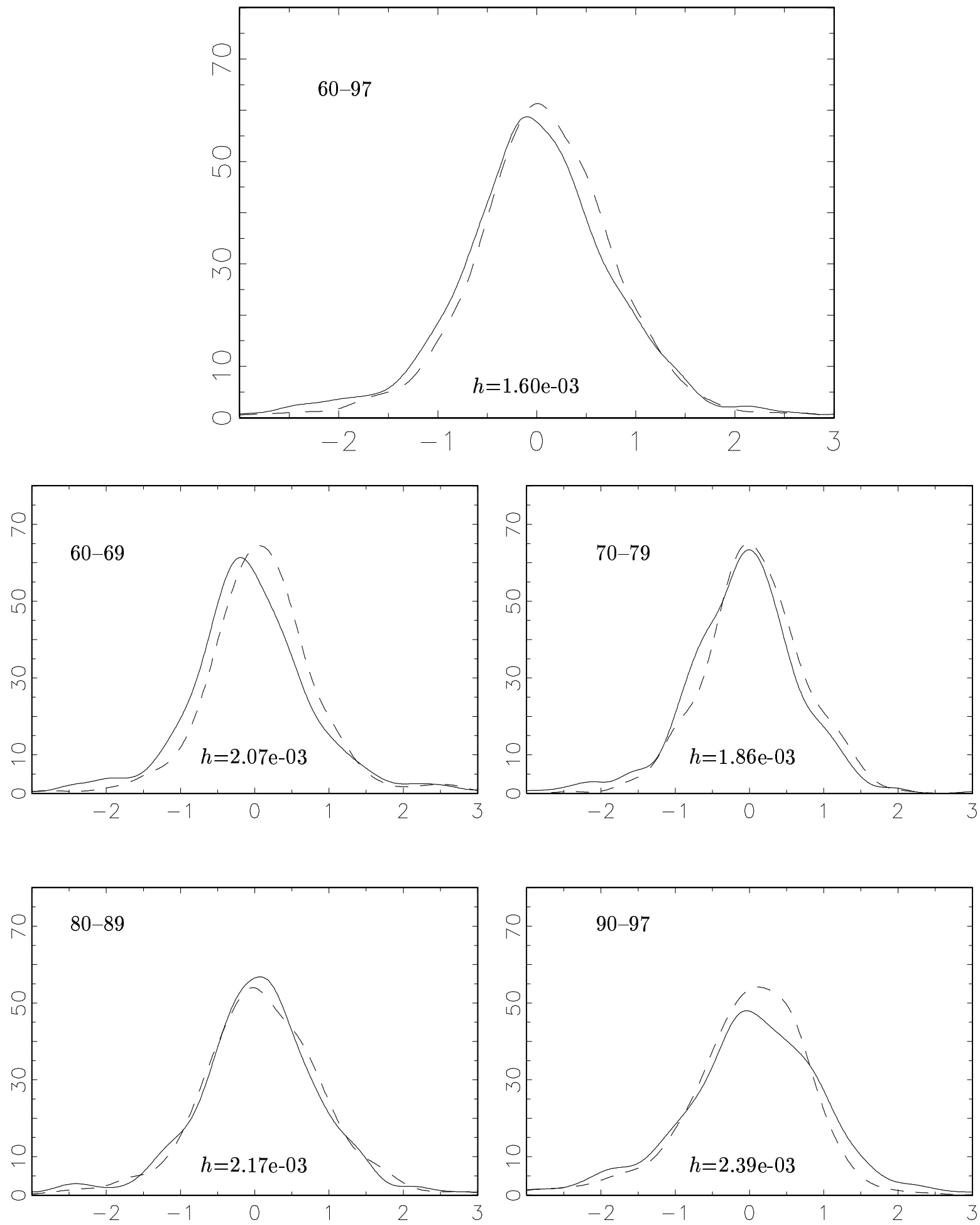

Figure 4: Estimated unconditional distribution of Monday $\hat{f}_{\text {mo }}(x)$ (solid lines) and Friday returns $\hat{f}_{\text {fr }}(x)$ (dashed lines). The relevant range of returns is $-0.03 \leq x \leq 0.03$. The scale of the $x$-axis is multiplied by $10^{2}$. 

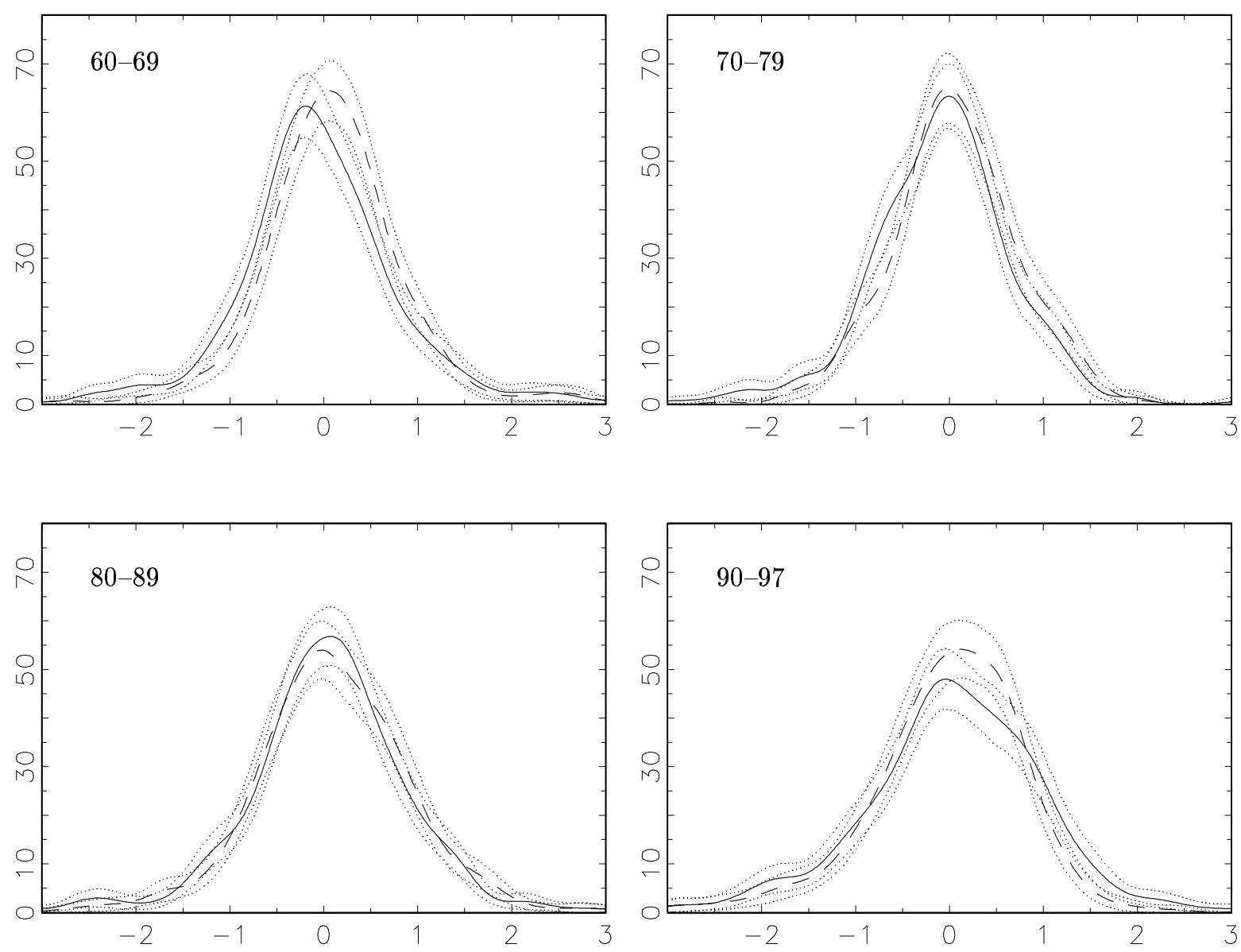

Figure 5: Estimated unconditional distribution of Monday $\hat{f}_{\mathrm{mo}}(x)$ (solid lines) and Friday returns $\hat{f}_{\mathrm{fr}}(x)$ with $95 \%$ confidence bands (dotted curves). The relevant range of returns is $-0.03 \leq x \leq 0.03$. The scale of the $x$-axis is multiplied by $10^{2}$. 